Revue

Revue de l'histoire des religions

de Ihistoire des religions

$3 \mid 2006$

Varia

\title{
Quelques usages du feu dans les rituels hittites et mésopotamiens
}

Several uses of fire in Hittite and Mesopotamian rituals

\section{Alice Mouton}

\section{OpenEdition}

\section{Journals}

Édition électronique

URL : http://journals.openedition.org/rhr/5167

DOI : $10.4000 /$ rhr.5167

ISSN : 2105-2573

\section{Éditeur}

Armand Colin

\section{Édition imprimée}

Date de publication : 1 septembre 2006

Pagination : 251-264

ISBN : 2200-92105-5

ISSN : 0035-1423

Référence électronique

Alice Mouton, "Quelques usages du feu dans les rituels hittites et mésopotamiens », Revue de l'histoire des religions [En ligne], 3 | 2006, mis en ligne le 25 janvier 2010, consulté le 10 décembre 2020. URL http://journals.openedition.org/rhr/5167 ; DOl : https://doi.org/10.4000/rhr.5167 


\author{
ALICE MOUTON \\ UMR 7044 "Étude des civilisations de l'Antiquité » \\ CNRS/Université de Strasbourg

\section{Quelques usages du feu dans les rituels hittites et mésopotamiens}

Nombreux sont les textes hittites qui mentionnent l'utilisation du feu sous la forme d'un foyer ou d'un flambeau lors des rituels magiques et thérapeutiques. Cet article tentera de répertorier les différentes vertus du feu dans le cadre de ces rituels. Trois principales fonctions seront examinées : le feu en tant que force destructrice, son statut d'agent purificateur et son rôle de voie de communication entre le monde divin et celui des hommes. Dans cette optique, des comparaisons seront faites avec les rituels et les incantations connus du monde mésopotamien.

\title{
Several uses of fire in Hittite and Mesopotamian rituals
}

A great number of hittite texts attest the fact that fire was used under the shape of a hearth or a torch during magical and therapeutic rituals. The aim of the following article will consist in listing the various powers of fire in the context of those rituals. Three functions will principally be examined: fire as a destroying force, its status of purifying agent and its role of passage between the divine world and the one of human beings. According to this point of view, several comparisons with rituals and incantations from the mesopotamian world will be provided. 
Il s'agit de tenter de définir les principaux usages que l'on fait du feu lors des cérémonies religieuses décrites sur les tablettes cunéiformes hittites (de l'Anatolie de la deuxième moitié du deuxième millénaire av. J.-C.).

Dans un souci comparatiste, il sera également question des témoignages provenant de la Mésopotamie, et plus particulièrement des textes néo-assyriens (du Nord de la Mésopotamie dans la première moitié du premier millénaire av. J.-C.), car ces documents nous offrent des données complémentaires à celles issues des textes hittites.

C'est presque exclusivement dans le cadre de rituels magiques et thérapeutiques que l'on a recours au feu. Faute de temps, les rituels de fumigation, c'est-à-dire ceux qui consistent à brûler une substance (encens ou certaines essences de bois) afin d'en tirer une fumée odorante souvent considérée comme purificatrice, ne seront pas étudiés ici. Les attestations de cette pratique sont très nombreuses aussi bien dans le monde hittite qu'en Mésopotamie, et cela fera l'objet d'une étude séparée.

Je ne mentionnerai donc que les utilisations du feu lorsque celui-ci apparaît sous la forme d'un foyer ou d'un flambeau ${ }^{1}$. Il faut d'ailleurs dès maintenant préciser que le foyer est considéré par les Hittites comme une divinité à part entière ${ }^{2}$.

Les aspects du feu qui seront étudiés plus particulièrement sont : 1) le feu destructeur ; 2) le feu purificateur et 3 ) le feu en tant que voie de communication entre le monde des dieux et celui des hommes.

\section{LE FEU DESTRUCTEUR}

Il arrive que l'on brûle des objets ou des animaux dans le but de les détruire. C'est en particulier le cas de certains substituts qui,

1. Ce thème est dorénavant survolé par le précieux ouvrage de Volkert Haas, Materia Magica et Medica Hethitica. Ein Beitrag zur Heilkunde im Alten Orient, Berlin-New York, 2003, qui nous offre une liste presque exhaustive des références hittites. Voir plus particulièrement V. Haas, Materia Magica..., p. 161-171.

2. Volkert Haas, Geschichte der hethitischen Religion, Handbuch der Orientalistik I/15, Leyde-New York-Cologne, 1994, p. 268. 
après avoir absorbé l'impureté de la personne pour laquelle le rituel de substitution est effectué, sont entièrement détruits par le feu. Dans ce cas, le feu sert probablement à éradiquer l'impureté de façon définitive.

Dans un rituel de substitution hittite destiné à protéger le couple royal, on lit (KUB 7.10 ii 1'-8') : «On brûle les substituts [du roi] dans un foyer: dans un foyer on immole un taureau et dans un (autre ?) foyer on immole un bélier et un bouc. Mais dans un (autre) foyer on brûle les substituts de la reine $: . .^{3}$ »

Il n'y a pas à ma connaissance d'incantation hittite qui décrive précisément le pouvoir qu'a le feu d'annihiler l'impureté ou ses sources. Cette lacune est comblée par le corpus mésopotamien, en particulier par la composition appelée Šurpu, «le fait de brûler ${ }^{4}$. Ce rituel, retranscrit sur au moins neuf tablettes, se décompose en toute une série de petits rituels successifs qui ont pour but ultime de purifier une personne tombée malade à la suite d'une attaque démoniaque. Celle-ci peut résulter d'une malédiction, d'une faute perpétrée par le malade (un parjure, la violation d'un tabou, etc.) notamment. Le rituel consiste principalement à jeter des offrandes alimentaires dans un brasier, après les avoir mises en contact physique avec le patient. Parmi ces denrées jetées au feu se trouve un oignon que le patient a préalablement porté sur lui, puis épluché. Or l'incantation qui accompagne ce geste rituel a été préservée. Elle indique la chose suivante (Tablette V-VI : 60-72) : «Incantation. Comme cet oignon qu'il pèle et jette au feu - le dieu Gira [= un des noms du dieu du feu] le consume entièrement - (oignon) [...] qui ne va pas être utilisé pour le repas des dieux ni des hommes, (de même) que le (par)jure, le serment (bafoué), la vengeance, le questionnement, la maladie, la détresse, la faute, le crime, le sacrilège, le manquement, la maladie qui est dans mon corps, (dans) ma chair, (dans)

3. Hans Martin Kümmel, Ersatzrituale für den hethitischen König, Studien zu den Boğazköy-Texten 3, Wiesbaden, 1967, p. 130 : [ $\breve{S} A$ LUGAL] tarpalius $<A N A>1$ GUNNI warnuanzi ANA 1 GUNNI-ma GU $_{4} \cdot$ MAH warnuwanzi $A N A<1>$ GUNNI-ma § UDU.ŠIR MÁŠ.GAL.ŠIR-ya warnuwanzi ANA 1 GUNNI-ma $\breve{S} A$ MUNUS.LUGAL tarpalliuš warnuwan[zi].

4. Édités par Erica Reiner, Šurpu. A Collection of Sumerian and Akkadian Incantations, Archiv für Orientforschung Beiheft 11, Graz, 1958. 
mes veines, (que tout cela) soit pelé comme cet oignon, que le dieu Gira les consume entièrement en ce jour, que le parjure s'éloigne et que moi je (re)vois la lumière ${ }^{5}$ ! » En ayant été en contact avec le corps du patient, l'oignon, ainsi que les autres denrées qui sont jetées au feu, a symboliquement absorbé la maladie de l'homme. Sa destruction par le feu entraîne, par le biais de la magie analogique, celle du mal que l'on veut combattre.

Le feu a donc le pouvoir d'anéantir le mal. Certaines incantations mésopotamiennes ${ }^{6} \mathrm{~s}^{\prime}$ adressent d'ailleurs directement au dieu du feu dont le nom varie selon les régions et les périodes : tantôt Gibil, tantôt Gira. Il faut préciser qu'un dieu du feu est également attesté dans les sources hittites où on l'appelle parfois Agni, nom manifestement hérité du monde indo-aryen.

D'autres rituels exorcistiques mésopotamiens, le plus souvent néo-assyriens, font appel au pouvoir destructeur du feu. C'est notamment le cas de divers rituels destinés à neutraliser un cauchemar ${ }^{7}$. Je ne citerai qu'un seul exemple (79-7-8, 77 Vo 18'-19') : « Si un homme a vu un mauvais rêve et qu'il est devenu inquiet, qu'il rac[onte] son rêve à un roseau tarītu ${ }^{8}$ puis qu'il brûle (le roseau) dans le feu. Il soufflera avec sa bouche (sur le feu). (Le mauvais rêve) sera dissous ${ }^{9}$. »

5. E. Reiner, Šurpu..., p. 31 : én kima sum.sar annī iqallapu-ma ana izi šub- $u$ dbil.gi qamû iqammû [...] ana naptan dingir $u$ lugal nu te-u nišu mamit turta mašaltu gig ta[ni]hi arni šerti gillati hițiti gig ša ina su.mu uzu ${ }^{\text {meš.mu }}$ $\mathrm{sa}^{\text {meš. }}$.mu gál-u gim sum.sar annī liqqalip-ma ina ume ann̄̄ giš.bar qamû liqmi mamit littași-ma anaku zalag 2 lumur.

6. Sally A. L. Butler, Mesopotamian Conceptions of Dreams and Dream Rituals, Alter Orient und Altes Testament 258, Münster 1998, p. 328. Certaines de ces incantations font allusion au feu en tant que force destructrice prenant possession du corps d'un individu. Il est alors le symbole de la fièvre (Wilfred G. Lambert, «Fire Incantations », Archiv für Orientforschung, 23, 1970, p. 39-45).

7. S. A. L. Butler, Mesopotamian Conceptions..., p. 185. J'aurai l'occasion de revenir plus en détails sur ce thème dans un autre cadre.

8. Roseau dont on a enlevé la couche superficielle vraisemblablement.

9. S. A. L. Butler, Mesopotamian Conceptions..., p. 332 : diš lú máš.ge 6

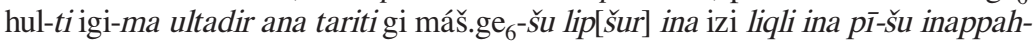
ma búr-i $i r]$. 


\section{LE FEU PURIFICATEUR}

Plusieurs rituels hittites attestent par ailleurs de l'utilisation du feu en tant qu'agent purificateur. La méthode employée alors varie : 1) soit on fait passer quelqu'un ou quelque chose entre deux foyers ou groupes de foyers ; 2) soit on le fait passer au-dessus ou à côté d'un seul foyer ; 3 ) soit encore on jette l'objet ou l'animal directement dans le foyer pour le purifier en le détruisant.

1. On peut utiliser deux foyers ou deux groupes de foyers pour former une aire de purification : un rituel brièvement décrit dans une tablette compilant plusieurs compositions (KUB 17.28 ${ }^{10}$ ) fait allusion à la pratique consistant à installer deux foyers ainsi que des substituts de part et d'autre d'un portail fait de matériaux périssables (une plante épineuse visiblement) et à faire passer les personnes qui doivent être purifiées au milieu. Les deux foyers semblent donc avoir pour fonction d'absorber l'impureté des personnes en question. L'effet supposé de ces foyers est renforcé par l'utilisation des substituts qui ont été préalablement coupés en deux : ces substituts sont un porcelet et un chiot, un bouc et un homme. Ce dernier est très vraisemblablement un prisonnier de guerre qui subit le même sort que les animaux-substituts mentionnés. L'utilisation de deux foyers miroirs, au milieu desquels passent une ou plusieurs personnes à purifier, est attestée dans d'autres compositions hittites telles qu'un rituel destiné à exorciser une malédiction (KBo 11.11 ${ }^{11}$ ). Au cours du rituel d'Ašhella d'Arzawa destiné à sauver une armée d'une épidémie, on retrouve cette même technique purificatrice dont bénéficient tous les soldats atteints de maladie (KUB 9.32+ et duplicats Vo 3-4) : « Ensuite, on fait brûler

10. Olivier Masson, « À propos d'un rituel hittite pour la lustration d'une armée : le rite de purification par le passage entre les deux parties d'une victime », Revue de l'histoire des religions, 137, 1950, p. 5-25.

11. Hans Gustav Güterbock et al. (éds.), The Hittite Dictionary of the Orient Institute of the University of Chicago (= CHD), Chicago, 1980 : voir CHD, vol. P, p. 12. 
un feu dans deux emplacements et on passe au milieu ${ }^{12}$. » Lors de la fête religieuse pratiquée pour la déesse Išhara à l'occasion du début du printemps, plusieurs gestes cathartiques sont combinés pour obtenir la purification de l'effigie divine (CTH 641, 2, B Ro 1-5 et duplicat KBo 21.42: 1-5) : "Quand on fait la fête du printemps pour Išhara, on fait ainsi : le devin place deux tables en vannerie et dépose dessus deux pains de soldats. Il retire la déesse et installe un feu de part et d'autre. Il verse de la farine sur le feu de part et d'autre et fait passer la déesse au milieu. On la lave (et) on l'oint (puis) il la replace sur la table ${ }^{13}$. » Un texte oraculaire (KUB 22.70 Vo 52-53) fait sans doute allusion à la même pratique, bien que de manière plus élusive : "Maintenant on fait passer cela [= les objets rituels] au milieu du bouc et du feu. Ensuite on le consacre et on le donne à la divinité de la même manière ${ }^{14}$. »

2. Passer des objets ou des animaux au-dessus ou à côté d'un foyer ou d'une torche: dans le rituel de naissance de Pāpanikri (KBo 5.1 iv 9-10) le prêtre patili chargé de la purification de la femme enceinte et de son bébé à venir «fait tourner» un agneau

12. Ali Dinçol, «Ašhella ritualı (CTH 394) ve Hititlerde salgın hastalıklara karşı yapılan majik işlemlere toplu bir bakış », Belleten 49/193, 1985, p. 1-40, et plus particulièrement p. 18 et $25:$ namma IZI 2 A $\breve{S} R A$ warnuanzi n-at-kan ištarna arha uwanzi.

13. Hans Gustav Güterbock, «Some Stray Boğazköy Tablets», dans Florilegium anatolicum. Mélanges offerts à Emmanuel Laroche, Paris, 1979, p. 137-144, et plus particulièrement p. 138 et 140 : mān ANA ${ }^{{ }^{D}}$ išhara EZEN $_{4}$ zenantaš DÙ-anzi nu kiššan DÙ-anzi LÚHAL 2 GIŠBANŠUR AD.KID dāi šerma-kan 2 NINDA.ERÍN.MEŠ dāi nu-kan DINGIR ${ }^{L U M}$ Šarā dāi nu IZI kēZ kēzziya dāi nu-kan ZÍD.DA ANA IZI kēz kēzziya išhūwai nu-kan DINGIR ${ }^{L U M}$ ištarna arha pēdai n-an arranzi iškiyanzi n-an-kan ANA GIŠBANŠUR EGIR-pa dāi. Il n'est pas impossible que l'on ait affaire ici à la purification et la reconsécration de l'effigie divine à l'occasion de la fête du printemps, mais le texte n'exprime pas clairement cette idée.

14. Ahmet Ünal, Ein Orakeltext über die Intrigen am hethitischen Hof (KUB XXII 70 = Bo 2011), Texte der Hethiter 6, Heidelberg, 1978, p. 94-95 : kinuna-at-kan MÁŠ́.GAL IZI-ya ištarna arha pēdanzi namma-at šuppiyahha $<n>z i$ $n$-at ANA DINGIR ${ }^{L I M}$ QATAMMA pianzi. Voir également Manfred Hutter, «Luwische Religion in den Traditionen aus Arzawa », dans Gernot Wilhelm (éd.), Akten des IV. Internationalen Kongresses für Hethitologie Würzburg, 4.-8. Oktober 1999, Studien zu den Boazköy-Texten 45, Wiesbaden, 2001, p. 224-234, et plus particulièrement p. 229. 
sept fois au-dessus de sept foyers. Ce procédé est combiné avec d'autres techniques purificatrices qui ont ici pour but de consacrer l'agneau qui servira probablement de substitut à l'enfant à naître ${ }^{15}$. Une pratique équivalente se retrouve dans un texte mésopotamien bilingue sumérien-akkadien (K. 1279 Vo 3-10), dans lequel on lit notamment: "Quand Kusu, le grand prêtre d'Enlil soulève l'encensoir et la torche, l'aspect lumineux (du dieu Gibil) brille dans l'obscurité. Marduk a prononcé la conjuration. On a fait bouger l'effigie divine au-dessus de lui $(=\mathrm{le} \text { feu })^{16}$. » Ce dernier geste semble avoir pour but de consacrer ou reconsacrer l'effigie d'une divinité. D'autres textes mésopotamiens pourraient être cités, dans lesquels un flambeau est manipulé lors d'un rituel de purification ${ }^{17}$.

3. La purification par la destruction : bien que les textes ne nous le disent pas clairement, il n'est pas impossible que la notion de purification ait un lien avec celle de destruction par immolation. En jetant un objet ou un animal dans un foyer, on neutralise les forces maléfiques qu'il renferme, ce qui revient à dire qu'on le purifie même si cette purification implique la destruction du contenant (l'objet ou l'animal servant souvent de substitut) tout comme du

15. Ferdinand Sommer et Hans Ehelolf, Das hethitische Ritual des Pāpanikri von Komana (KBo V 1 = Bo 2001). Text, Übersetzungsversuch, Erläuterungen, Boghazköi-Studien 10, Leipzig, 1924, p. 12*-13*: n-ašta Lú patiliš SILA ${ }_{4}$ anda pēdāi n-an-kan ANA 7 IZI 7-ŠU šer arha wahnuzi. « Le patili- amène l'agneau et il le fait tourner sept fois au-dessus de sept foyers. » Je suis actuellement en train de finir une étude sur les rituels de naissance kizzuwatniens, étude dans laquelle j'analyse en détails les gestes rituels qui sont mentionnés ici.

16. Giovanni Conti, «A proposito di Gibil, dio del fuoco », dans Simonetta Graziani (éd.), Studi sul Vicino Oriente antico dedicati alla memoria di Luigi Cagni I, Naples, 2000, p. 125-135, et plus particulièrement p. 129-130 : version sumérienne: ${ }^{[\mathrm{d}]} \mathrm{kù.sù} \mathrm{sanga} \mathrm{a}_{4} \cdot \mathrm{mah}\left[{ }^{\mathrm{d}} \mathrm{e}\right] \mathrm{n}$.líl.lá.k[ $\mathrm{e}_{4}$ níg.n]a gi.izi.lá ba.ni.íb.è múš.me.bi dádag.ga.ke ${ }_{4}$ kúkku.ga dádag [ab].gá.gá dasar.lú.hi

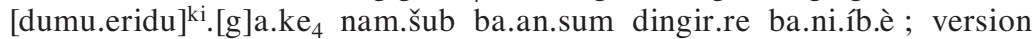
akkadienne : ${ }^{\mathrm{d}} \min$ min-hu ša ${ }^{\mathrm{d}} \min \min \min -u$ ušbi' îma zîmê-šu namrûtu unammaru ik[le]ti marduk mâr eridu šiptam iddi dingir ušbi'ušu.

17. Stefan Maul, Zukunftsbewältigung. Eine Untersuchung altorientalischen Denkens anhand der babylonisch-assyrischen Löserituale (Namburbi), Baghdader Forschungen 18, Mainz, 1994, p. 95. 
contenu (l'impureté ou tout autre élément néfaste) ${ }^{18}$. Le témoignage allant le plus clairement dans ce sens est une incantation insérée dans une des versions du mythe de Telipinu (KUB 17.10 iii 8-16) : «J'ai fait brûler (un feu) pour Telipinu ici et j'ai fait brûler (un feu) là. J'ai pris du corps de Telipinu son mal, j'ai pris sa faute, j'ai pris sa colère, j'ai pris son courroux, j'ai pris le warku-, j'ai pris la fureur. Telipinu est en colère. Son esprit et son karat- sont comprimés comme des broussailles. Tout comme on a brûlé ces broussailles, que la colère, le courroux, la faute (et) la fureur de Telipinu brûlent de la même manière ${ }^{19}$. » La purification de Telipinu se fait donc par la destruction par le feu des maux qui le hantaient. Un autre exemple peut être cité ${ }^{20}$, dans lequel un ornithomancien qui a prononcé une «mauvaise parole » devant une divinité doit essuyer sa bouche avec une sorte de gâteau, gâteau qui sera par la suite jeté dans le feu. Le gâteau a donc absorbé la mauvaise parole de l'homme en entrant en contact direct avec la bouche de celui-ci. Par la suite, le

18. Voir dans le même ordre d'idées, les brèves réflexions émises par Piotr Michalowski, «The Torch and the Censer », dans Mark E. Cohen et al. (éd.), The Tablet and the Scroll. Near Eastern Studies in Honor of William W. Hallo, Bethesda, 1993, p. 152-162, et plus particulièrement p. 157.

19. Emmanuel Laroche, Textes mythologiques hittites en transcription, Paris, 1965, p. 34-35 et Harry A. Hoffner, Hittite Myths, $2^{\text {nd }}$ ed., Society of Biblical Literature 2, Atlanta, 1998, p. 16: nu D telipinui šêr arha duwān

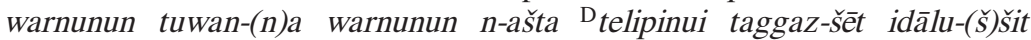

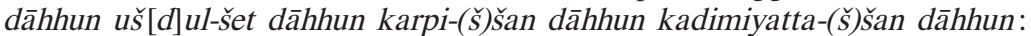

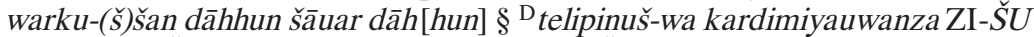
karā [z-Šiš ${ }^{\text {GIŠ }}$ waršammaš uišūriyatati nu kūš GIŠ warš[ammuš] māhhan warnuwer D telipinuwaš-(̌̌)a karpi[š] kardimiyaz waštul šāuar QATAMMA warā[nu].

20. KBo 17.105 ii 34'-42' : mān LÚMUŠEN.DÙ kuiški PANIDINGIR ${ }^{\text {LIM }}$ idālu uttar memian harzi n-aš-ma-at-kan kardimanuwan kuiški harzi § n-ašta LÚ.MEŠMUŠEN.DÙ-ya UZUKAxU-ŠUNU IŠTU NINDA.İ.E.DÉ.A arha anšanteš ašandu nu NINDA.İ.E.DÉ.A kuiš ANA DLAMMA KUŠ kuršaš peran kitta $n$-an MUNUSŠU.GI šarā dāi n-an-kan anda hašš̄ peššiēzzi ANA LÚ.MEŠMUŠEN.DÙya-kan kuiš NINDA.İ.E.DÉ.A KAxU-išmi kitta n-an-za parā danzi n-an-kan anda haššī peššiyanzi. «Si un ornithomancien a prononcé une mauvaise parole en présence de la divinité, il a provoqué la colère (de la divinité). § Que les ornithomanciens aient leur bouche essuyée avec du gâteau de graisse. Le gâteau de graisse qui a été posé devant le dieu tutélaire du sac de chasseur, la Vieille Femme le prend et le jette dans le foyer. Le gâteau de graisse qui se tenait pour la bouche des ornithomanciens, ils le prennent et le jettent dans le foyer. » 
gâteau - et la mauvaise parole avec lui - est purifié par le feu. Le fait que ce soit un comestible qui ait été choisi pour tenir cet office pourrait indiquer que le gâteau purifié par le feu sert secondairement d'offrande alimentaire à la divinité que l'ornithomancien a offensée. Cela pourrait également être le cas de l'oignon manipulé dans le rituel mésopotamien Šurpu qui a été mentionné auparavant. Il s'agit cependant de rester prudent quant à ce dernier aspect du rituel, les textes ne confirmant pas cette hypothèse.

Le rôle purificateur du feu est également bien attesté dans le monde mésopotamien, comme en témoigne notamment une incantation composée en langue sumérienne en l'honneur de cette divinité (YOS 11.59) : «Incantation. Gibil, splendeur, Gibil, paré de splendeur, Gibil, celui qui purifie, celui qui fait resplendir, c'est lui qui rend le ciel pur, c'est lui qui rend la terre pure [le texte fait ensuite allusion au pouvoir cathartique de la fumigation] [...]. Gibil, lumière du ciel et de la terre, tu es celui qui rénove et purifie

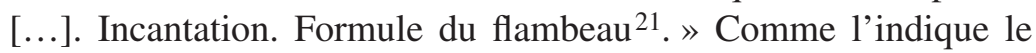
titre de cette incantation, celle-ci devait être prononcée en même temps qu'un flambeau était manipulé dans le but de purifier un objet ou un être vivant.

\section{LE FEU COMME VOIE DE COMMUNICATION ENTRE LE MONDE DES DIEUX ET CELUI DES HOMMES}

Dans au moins une région de l'Anatolie hittite, à savoir le Kizzuwatna (au sud sud-ouest de l'Anatolie), le feu était manifestement vu comme un passage entre les deux mondes existant: le monde invisible des entités surnaturelles (dieux, démons et esprits) et celui des hommes.

1. Au Kizzuwatna en effet, il est fréquent que l'on brûle entièrement un animal pour le transmettre aux dieux. Dans le rituel de naissance de Pāpanikri (KBo 5.1 ii 2), on effectue cette pratique

21. G. Conti, «A proposito di Gibil...», p. 125-126 : én.é.nu.ru d gibil 6 še.er.zi ${ }^{d}$ gibil $_{6}$ še.er.gán d ${ }^{\text {gibil }}{ }_{6}$ lú sikil lú dadag.ge an kù.ge.dam ki sikil.e.dam

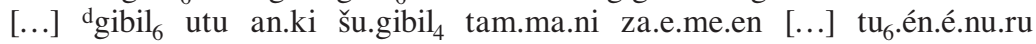
inim.inim.ma gi.izi.lá.kam. 
«pour (expier) l'offense (et) la faute ${ }^{22}$ ». L'offrande ambašši à laquelle est associée l'offrande keldi consiste précisément à immoler entièrement un animal ${ }^{23}$. Or cette pratique est très bien documentée dans les textes religieux provenant du Kizzuwatna alors qu'elle semble absente du reste de l'Anatolie hittite. Elle pourrait en outre avoir été héritée de la Syrie septentrionale. Dans un texte décrivant un problème d'élocution étant survenu au roi hittite Muršili II (KUB 48.100 + Ro 11-12), on écrit : «Il a été déterminé (par oracle) qu'il faut lui [= la divinité] envoyer un bœufsubstitut, qu'il faut [l']entourer avec du feu et qu'il faut entourer avec du feu des oiseaux ${ }^{24}$. » Dans d'autres cas, des offrandes alimentaires sont jetées dans le feu, sans doute pour la même raison: on peut mentionner du fromage (KUB 9.28 ii 3-425), de l'huile et la boisson memal (KBo 11.32 Ro 13).

2. Une composition provenant elle aussi du Kizzuwatna indique que les dieux peuvent eux-mêmes utiliser le feu comme une voie d'accès au monde des mortels. Il s'agit d'un rite d'évocation (KUB 15.34 iii 56-57) dans lequel on lit: «Ensuite, il [= le devin] attire (les dieux) par le feu de la même manière et [dit] : "Si (vous), dieux masculins du cèdre, (êtes) dans le feu qui brûle", etc. ${ }^{26} \gg$ Dans

22. F. Sommer et H. Ehelolf, Das hethitische Ritual..., p. 6*-7*: nu ENMEŚ SÍSKUR šinapšiya pānzi nu 2 MUŠEN haratni wašduli warnuwanzi. «Les commanditaires du rituel vont au šinapši- et ils immolent deux oiseaux pour l'offense (et) la faute. »

23. V. Haas, Geschichte..., p. 661-664 et Daniel Schwemer, « Das alttestamentliche Doppelritual 'lwt wšlmym im Horizont der hurritischen Opfertermini ambašši und keldi », Studies on the Civilization and Culture of Nuzi and the Hurrians 7, 1995, p. 81-116.

24. René Lebrun, « L'aphasie de Mursili II = CTH 486 », Hethitica 6, 1985, p. 103-137, et plus particulièrement p. 104 et $110: n u-(\check{S}) \check{S} i \mathrm{GU}_{4}$ pūhugariš pīyauwanzi IZI-it wahnumanzi MUŠEN ${ }^{H A ́}$ wahnummanzi SIxSÁ-at.

25. Cité par Harry A. Hoffner, «A Native Akkadian Cognate to West Semitic *GBN "Cheese" ? », Journal of the American Oriental Society 86, 1966, p. 27-31, et plus particulièrement p. 28.

26. Volkert Haas et Gernot Wilhelm, Hurritische und luwische Riten aus Kizzuwatna, Hurritologische Studien I, Alter Orient und Altes Testament Sonderreihe 3, Neukirchen-Vluyn, 1974, p. 200 : EGIR- $\breve{S} U$-ma pahhuenaz QATAMMA huittiyaz[i nu tezzi] mān-za DINGIR MEŠ LÚMEŠ GIŠERIN-aš waranti pahhu[eni]. 
cette incantation, le praticien envisage ici les différents lieux dans lesquels les dieux peuvent se manifester aux hommes, formule qui est bien connue dans la documentation religieuse hittite. Mais il est important de remarquer que seul ce texte d'origine kizzuwatnienne mentionne le feu dans un tel contexte, et ce n'est sans doute pas l'effet du hasard.

\section{QUelques Passages AMBiguS}

Dans d'autres cas, il n'est pas vraiment possible de savoir pour quelle raison précise le feu est employé. Dans une fête religieuse kizzuwatnienne (KUB 23.28+ i 25'-27'), on indique : « Le lendemain, dans la maison des grands-pères on entoure les [divinités] du père avec du feu ${ }^{27}$.» La fonction de ce geste rituel n'étant pas explicitée dans le texte, il est difficile de donner une interprétation définitive de ce passage. Peut-être a-t-on affaire ici à une reconsécration par le feu des effigies des divinités du père, divinités dont l'identité précise reste quelque peu énigmatique. Le feu endosserait alors une fonction cathartique à l'instar des autres textes déjà étudiés.

Dans un rituel exorcistique (rituel de Maštigga), on flanque sept stèles de deux foyers ${ }^{28}$. Il semble que ces foyers traitent magiquement les stèles, bien qu'il soit difficile de préciser comment. Une incantation est en effet prononcée à la suite du texte, dans laquelle on fait allusion au fait que les stèles sont sur le point de tomber ${ }^{29}$.

27. Ilse Wegner et Mirjo Salvini, Die hethitisch-hurritischen Ritualtafeln des (h)išuwa-Festes, Corpus der hurritischen Sprachdenkmäler I/4, Rome,

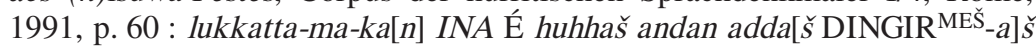
pahhuenit wahnuanzi.

28. KBo 39.8 iii 54 : [n] u pahhur ZAG-az GÙB-laz-(zi)ya warnuwanzi

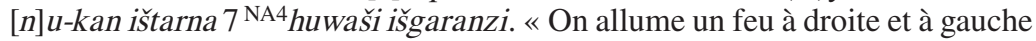
et on installe sept stèles huwaši au milieu. » (Jared L. Miller, Studies in the Origins, Development and Interpretation of the Kizzuwatna Rituals, Studien zu den Boğazköy-Texten 46, Wiesbaden, 2004, p. 97.)

29. J. L. Miller, Studies in the Origins..., p. 99. 


\section{DES MYTHES DU FEU}

À côté des gestes rituels employant le feu, plusieurs incantations et récits mythologiques se réfèrent à lui. Une tablette compilant plusieurs compositions hittites retranscrit un récit mythologique où le rôle principal est tenu par le feu (KUB 17.8 iv). Dans ce texte où mythe, incantation et gestes rituels se mêlent, c'est avant tout au pouvoir destructeur du feu que l'on fait allusion. Le passage illustrant le plus clairement ce propos est le suivant (iv 7-14) : «Que la maladie de sa tête devienne fumée et qu'elle parte dans le ciel ! Que la Terre Noire [= le monde souterrain] emporte la maladie hors de sa main ! Le nuage ne parvient pas à bout de la maladie. Que d'en haut le ciel en vienne à bout ! Que d'en bas la Terre Noire en vienne à bout ! Conjuration du feu. $\S$ On donna (au feu) : on lui donna les grains, on lui donna la maladie, on lui donna la maladie des yeux, on lui donna la maladie de la main, on lui donna la maladie de la tête ${ }^{30}$. » Le feu semble bien être l'agent destructeur de la maladie, agent représentant les forces du ciel et du monde souterrain. Le reste du texte recourt à un topos littéraire connu dans d'autres compositions mythologiques hittites, à savoir l'extinction d'un foyer. Dans ce type de contextes, le foyer est vu comme le centre de l'habitat des hommes, l'élément indispensable à leur vie de tous les jours, puisqu'il sert à la fois à cuire les aliments et à chauffer les pièces de la maison. C'est pourquoi l'extinction d'un foyer symbolise le plus souvent l'extinction d'une maisonnée résultant de l'abandon de ses habitants par les

30. Galina Kellerman, « KUB XVII 8 IV : un mythe du feu », Hethitica 8 , 1987, p. 215-235, et plus particulièrement p. 217 et 219 : nu-wa haršanaš-šan GIG-aš kammarāš kišaru n-at nepi[š]a paiddu IŠTUŠU-ma-kan GIG-ŠUGE iš KI-anzipaš karapdu nu alpaš GIG-an ÜL tarahzi n-an-za šer nepišanza tarahdu kattan-(n)a-za GE 6 -iš KI-aš tarahdu ŠIPAT IZI-naš § pīer nu-(̌̌s)̌si halkiyaš

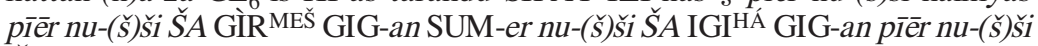
$\breve{S} A$ SAG.DU GIG-an piēr. 
dieux. Cet abandon est lui-même ressenti par les Hittites comme une manifestation de la colère divine ${ }^{31}$.

À côté de ce texte, il faut en citer un deuxième (KUB 43.62) ${ }^{32}$, dont le genre est tout à fait analogue à celui du précédent, à savoir un mélange de rituel de guérison et de récit mythologique sur le feu. Le récit lui-même - d'ailleurs qualifié de conjuration dans le texte ${ }^{33}$ - est cependant très différent de celui que nous avons vu auparavant. Cette fois-ci, il semble que le feu divinisé, décrit comme le fils du dieu Soleil, ait décidé de s'en aller après s'être mis en colère pour une raison que nous ignorons. Cette description rappelle les mythes anatoliens des dieux disparus, dans lesquels une divinité dont l'identité varie décide après un coup de colère d'abandonner toutes ses responsabilités et de partir se cacher.

\section{ConCLusion}

Aussi bien chez les Hittites que chez leurs voisins mésopotamiens, le feu est abondamment employé lors des rituels magiques et thérapeutiques. Son effet purificateur est lié à la force destructrice qui le caractérise. Cette puissance tantôt bienfaisante tantôt maléfique est d'ailleurs personnifiée sous la forme d'un dieu du feu aussi bien en Mésopotamie qu'en pays hittite.

31. $C f$. dans le mythe de Kešši, un des rêves du chasseur décrit les divinités du père de ce dernier qui s'efforcent d'entretenir un foyer sur le point de s'éteindre. Dans les mythes des dieux disparus, les foyers des mortels s'éteignent tout comme l'ensemble de leurs ressources. Pour une traduction et une bibliographie récentes pour l'ensemble de ces mythes, voir H. A. Hoffner, Hittite Myths.

32. Ahmet Ünal, « Parts of Trees in Hittite according to a Medical Incantation Text (KUB 43.62)», dans Heinrich Otten et al. (éds.), Hittite and Other Anatolian and Near Eastern Studies in Honour of Sedat Alp, Anadolu medeniyetlerini araştırma ve tanıtma vakfı yayınları 1, Ankara, 1992, p. 493-500 et H. Craig Melchert, «Hittite antaka- "loins" and an Overlooked Myth about Fire », dans Gary Beckman et al. (éds.), Hittite Studies in Honor of Harry H. Hoffner Jr. on the Occasion of His 65th Birthday, Winona Lake, 2003, p. 281-287.

33. D'autres conjurations du feu sont d'ailleurs mentionnées dans les sources hittites : dans KBo 22.107 i 11 (pahhur huekmi: "Je conjure le feu. ») et dans KBo 22.102 Vo? 4' ([D]UB.1.KAM ŠIPAT pah[hur] : «Une tablette : conjuration du fe[u] »). 
Quelques textes hittites provenant plus spécifiquement du Kizzuwatna indiquent en outre que le feu peut servir de passerelle entre le monde des hommes et celui des dieux. Le fait d'immoler des victimes sacrificielles est alors considéré comme un moyen de transmettre ces victimes aux dieux. Cette conception rappelle quelque peu celle qui avait cours dans la Grèce ancienne, où les viandes sacrificielles étaient elles aussi brûlées pour que les divinités puissent se repaître du fumet produit par leur combustion ${ }^{34}$.

alicemouton@hotmail.com

34. Cette contribution a été lue lors du VI ${ }^{\mathrm{e}}$ séminaire interdisciplinaire des Instituts d'archéologie de l'Université Marc Bloch (Strasbourg) intitulé «L'archéologie du feu » et organisé du 9 au 11 Janvier 2006 par le professeur J.-J. Schwien. 